\title{
Le siège métropolitain de Québec et la naissance de la Confédération
}

\section{Armand Gagné}

Volume 34, 1967

URI : https://id.erudit.org/iderudit/1007420ar

DOI : https://doi.org/10.7202/1007420ar

Aller au sommaire du numéro

Éditeur(s)

Les Éditions Historia Ecclesiæ Catholicæ Canadensis Inc.

ISSN

0318-6172 (imprimé)

1927-7067 (numérique)

Découvrir la revue

Citer cet article

Gagné, A. (1967). Le siège métropolitain de Québec et la naissance de la Confédération. Sessions d'étude - Société canadienne d'histoire de l'Église catholique, 34, 41-54. https://doi.org/10.7202/1007420ar

Tous droits réservés @ Les Éditions Historia Ecclesiæ Catholicæ Canadensis Inc., 1968
Ce document est protégé par la loi sur le droit d'auteur. L'utilisation des services d'Érudit (y compris la reproduction) est assujettie à sa politique d'utilisation que vous pouvez consulter en ligne.

https://apropos.erudit.org/fr/usagers/politique-dutilisation/ 


\section{Le siège métropolitain de Québec et la naissance de la Confédération}

Il n'est peut-être pas sans intérêt de s'interroger sur les positions du plus ancien diocèse du Canada au moment où les destinées de ce pays allaient connaître un nouveau départ. Appelé jusqu'en 1852 le diocèse du Canada, l'archidiocèse de Québec continue d'être à l'époque de la Confédération le carrefour de la vie religieuse au pays, malgré les multiples démembrements de territoire qui en 1867 avaient porté à dixneuf le nombre des diocèses de Terre-Neuve à Victoria. Dix de ces diocèses se trouvent alors sous la juridiction de Québec comme suffragants,

Dans l'histoire des événements qui, de 1864 à 1867, ont donné naissance à la Confédération, on ne voit pas que le siège métropolitain de Québec ait cherché des tribunes pour définir sa position qui, pourtant, dès le mois de juin 1864, se trouve déjà clairement établie et ne changera pas d'un iota par la suite. Cette discrétion s'explique pour plusieurs raisons. L'une d'elles est sans contredit l'épreuve de maladie dont fut atteint en 1855 l'archevêque de Québec, Mgr Turgeon, et qui devait priver le siège métropolitain de son chef jusqu'en 1867, si bien que le coadjuteur Mgr Baillargeon, nommé aussitôt administrateur, se trouva pendant cette longue période dans une position en quelque sorte inférieure à celle de ses suffragants.

On se surprend d'autre part que le projet de confédération ait été accueilli aussi spontanément par l'archevêché de Québec, sans nulle trace d'appréhension ou de panique, comme si les implications du nouveau régime avaient déjà été sereinement étudiées et évaluées. C'est que parmi les collaborateurs et conseillers de Mgr Baillargeon se trouvaient deux hommes dont l'histoire religieuse du $\mathrm{XIX}^{\circ}$ siècle a gardé la marque : d'une part M. E.-A. Taschereau, recteur de l'Université Laval et futur archevêque de Québec; d'autre part, M. C.-F. Cazeau, Grand Vicaire de Québec pendant plus de trente ans. Sans ce dernier, la Confédération n'aurait peut-être pas été ce qu'elle a été. Consulté sur tous les sujets par l'épiscopat dont il était une figure familière il avait présidé à la fondation de la plupart des nouveaux diocèses il entretenait des contacts étroits avec la hiérarchie sociale, juges, ministres, représentants du peuple, fonctionnaires publics, et souvent hommes les plus opposés d'opinions et de croyances. On ne peut douter que, confident de longue date des G.-E. Cartier, E.-P. Taché et H. Langevin, il ait été mêlé de très près au projet de confédération. On s'explique mieux dans cette perspective que le projet n'ait causé aucune surprise à l'archevêché de Québec et qu'au contraire, la conviction des chefs religieux ait été aussi rapidement et aussi fermement acquise au projet d'une nouvelle constitution. 
Tous les sièges suffragants de Québec ne partageaient pas, du moins au départ, une opinion aussi sereine. C'est dans les échanges répétés avec ces diocèses que le siège métropolitain de Québec fut appelé à définir ses positions, aussi bien qu'à les maintenir, comme le laisse voir le déroulement des événements de l'époque.

\section{PÉRIODE PRÉ-CONFÉDÉRATIVE}

A partir du moment où le projet de confédération se trouve résolument engagé jusqu'à la sanction de la loi en mars 1867, il est possible de distinguer trois phases, assez révélatrices de l'attitude du siège métropolitain de Québec dans la genèse de la nouvelle constitution. La première phase origine avec l'alliance de George Brown et s'échelonne jusqu'à la Conférence de Québec. La deuxième s'étend de la Conférence de Québec jusqu'au retrait du Bill sur l'éducation en août 1866. Enfin la troisième, marquée principalement par la Conférence de Londres, se termine avec la sanction royale du 29 mars 1867.

Aussitôt connue l'alliance de George Brown avec le tandem CartierMacdonald, les réactions se font vives, principalement à Montréal, et elles ont tôt fait de se répercuter à Québec. L'alerte est donnée par Mgr Bourget le 28 juin 1864, précédée et suivie de l'écho de George Clerk, rédacteur du True Witness, les 26 et 29 juin. Mgr Bourget, consterné par l'alliance de Brown, écrit au Grand Vicaire Cazeau que, si c'est vraiment la seule planche de salut, il faut se préparer au naufrage ${ }^{1}$. Comme un bon sujet doit obéir à son gouvernement, il obéira lui aussi au nouveau gouvernement, quoiqu'il ne lui inspire aucune confiance. Pour le moment, il entend se limiter au silence, attendant les faits. Mais, si plus tard, les intérêts du catholicisme sont en danger, il fera toute l'opposition que peut faire un évêque à un gouvernement qui oublie ses devoirs.

La réponse de $\mathrm{M}$. Cazeau à Mgr Bourget n'ayant pas été conservée, c'est par celle qu'il fit à Clerk le $1^{\text {er }}$ juillet ${ }^{2}$ qu'il nous sera possible de connaître pour la première fois la position de l'archevêché de Québec. A Clerk, saisi lui aussi d'une sorte d'affolement, M. Cazeau avoue qu'il partage ses craintes et qu'il se laisserait aller au découragement, s'il n'avait la plus grande confiance dans les hommes qui représentent le Bas-Canada dans le Ministère, surtout E.-P. Taché et G.-E. Cartier. Mais, étant donné que le gouvernement était devenu impossible et que de nouvelles élections risquaient d'amener la représentation basée sur la population, le moyen de conjurer le danger était de s'occuper du projet de fédération qui donnerait au Bas-Canada le droit de se gouverner par lui-même. D'autant plus que les "clear-grits " n'ayant que trois voix contre neuf conservatrices dans le Ministère, l'alliance de Brown n'a pas de quoi inquiéter comme en 1848 , alors que les ministres bas-canadiens " marchaient à la queue " de ce dernier. Quant aux ministres du Bas-Canada, il déclare qu'ils n'ont rien d'arrêté au sujet du projet

1 Diocèse de Montréal, XI, 62.

2 Correspondance de G. Clerk [liasse non inventoriée]. 
de fédération devenu pour ainsi dire inévitable; mais il faut être assuré qu'ils ne négligeront rien pour que le Bas-Canada ait toutes les garanties que l'on puisse désirer. En attendant, les ministres souhaitent que le projet soit discuté avec la plus grande liberté possible dans les journaux, afin que la lumière se fasse.

Dans la réponse de $M$. Cazeau se trouve déjà définie sans équivoque la position du siège de Québec, position qu'il maintiendra et défendra contre vents et marées. M. Cazeau semble avoir tout pesé du nouveau régime, les implications politiques comme les implications religieuses. Quant à celles-là, la Confédération est la seule solution possible; quant à celles-ci, sa confiance repose sur des " hommes dévoués à l'Eglise et qui ont toujours été ses plus fermes défenseurs ».

Entre temps, les évêques de la province ecclésiastique de Québec sont convoqués à leur assemblée plénière, devant d'abord se tenir à Kingston, mais reportée au 18 octobre 1864 et à Trois-Rivières, pour célébrer à la même occasion le jubilé d'or de Mgr Cooke. La première question à l'ordre du jour, et proposée par les évêques de Montréal et de Sandwich, se lit comme suit : "Quelle conduite tenir, et quelle direction proposer au clergé et au peuple dans la présente crise ministérielle $^{3}$ ?" Un peu plus loin, la dixième question, proposée par $\mathrm{Mgr}$ Baillargeon lui-même, montre que ce dernier n'est pas uniquement préoccupé par le projet de confédération : «Le Coadjuteur de l'Archevêque de Québec, ne pouvant avoir un coadjuteur lui-même, ne ferait-il pas bien de prier le Saint-Siège d'accepter sa démission et de nommer un autre coadjuteur ${ }^{4}$ ?"

Il serait du plus grand intérêt de parcourir le procès-verbal de cette réunion, s'il en existait un. Mgr Baillargeon a, de son côté, fait parvenir le 27 octobre un long compte rendu de ces assises au cardinal Barnabo, mais il ne dit pas un mot des discussions sur le projet de confédération. Tout n'est pas perdu pour autant, puisque l'on sait qu'à l'automne 1864, il y a eu consultation au sein de l'épiscopat sur le projet de confédération. Il est vraisemblable de penser qu'à la même occasion on se soit entendu pour respecter la consigne du silence sur la question de la confédération. Quant à la démission de Mgr Baillargeon, que ses suffragants ont refusé d'appuyer, ce n'est que la première de nombreuses démarches faites en ce sens auprès du Saint-Siège, lesquelles se termineront dans un tête-à-tête avec Pie IX lors du $1^{\text {er }}$ Concile du Vatican. Le Pape aura ces paroles : "Moi aussi, je suis vieux; comme vous, j'ai des infirmités et plus que vous je suis affligé, et cependant je mourrai ... les armes à la main; mourez donc vous aussi sur le champ de bataille ${ }^{6}$." Revenant à la charge auprès du cardinal

3 Reg. Lettres, 28, 166.

4 Ibid.

5 Les évêques de Saint-Boniface et de Sandwich, n'ayant pu être présents à la réunion de Trois-Rivières, devaient recevoir le compte rendu de ces assises, du moins Mgr Taché, comme le lui affirme Mgr Baillargeon dans une lettre du 23 septembre. Rien n'indique toutefois que ce dernier ait tenu promesse.

6 Mgr H. Têtu, Les Evêques de Québec, p. 639. 
Barnabo dans la lettre déjà citée du 27 octobre, Mgr Baillargeon invoquera pour motiver sa démission cinq raisons dont la deuxième est étroitement reliée à la crise politique de l'époque :

$2^{\circ}$ Mon inaptitude absolue pour les affaires politiques, dans un temps de crise comme celui que nous traversons en Canada, où il faudrait à la tête du clergé un homme capable de guider ses opinions et diriger son action par rapport aux changements qui s'opèrent dans la constitution de notre législation, et d'exercer une salutaire influence sur les hommes d'état pour le maintien des droits de l'église et la défense de la religion.

Cette confidence pourrait surprendre, voire même inquiéter si nous ne connaissions déjà le tempérament de $\mathrm{Mgr}$ Baillargeon et le relief qu'il peut donner dans cette lettre aux motifs de sa démission ? Néanmoins, il ressentira cette même inappétence au moment de rédiger son mandement sur la Confédération et il faut croire par ces indices que la politique n'avait pour lui rien de trop complaisant.

Depuis juin 1864, des événements aussi importants que les Conférences de Charlottetown et de Québec se sont succédé et le projet de confédération fait son chemin. Lors de la Conférence de Québec, l'Université Laval avait, pour souligner l'événement, invité les délégués à une réception d'honneur le 20 octobre. On ne voit pas que l'archevêché de Québec y ait été représenté. Le texte d'une adresse lue par M. E.-A. Taschereau et celui de la réponse des délégués - le tout dans un style très conventionnel - nous ont été conservés ${ }^{8}$.

L'épiscopat, déjà préoccupé par la crise ranimée du Code Civil, s'inquiète maintenant de la teneur d'un article relatif au mariage et au divorce dans la nouvelle constitution ${ }^{9}$. C'est la deuxième phase de cette période pré-confédérative, marquée par une sorte de controverse entre évêques et théologiens, au sein de laquelle le siège métropolitain de Québec défendra, malgré la résistance de certains de ses suffragants,

7 D'origine insulaire et de tempérament effacé, gravement atteint au surplus par les privations de ses jeunes années, Mgr Baillargeon se sentira inférieur à la tâche jusque sur son lit de mort. Pourtant, son œuvre, encore mal connue, montre à l'évidence qu'il savait redoubler d'énergie devant les difficultés. On est étonné de son tact et de sa délicatesse à l'égard des personnes, de sa clairvoyance et de sa sérénité dans la solution des graves problèmes, tant intérieurs que supradiocésains, qui s'ajoutaient à la crise politique de l'heure: codification de nos lois, choix de nouveaux évêques, gaumisme, morcellement du diocèse, projet d'université à Montréal, incendies, choléra, etc.

8 ASQ, Université 103 , nos 89 et $89 a$.

9 Il semble superflu de reprendre ici l'exposé de cette question, déjà résumée par le R.P. Léon Pouliot, S.J. dans son étude $\mathbf{M g r}$ Bourget et la Contédération : - Une clause du projet de confédération enlevait aux gouvernements provinciaux pour les confier au gouvernement fédéral toutes les questions relatives au mariage et au divorce. Pour les Provinces Maritimes, où le divorce était déjà reconnu par la loi, il s'agissait d'un simple transfert de juridiction. Pour les Canadas-Unis, où le divorce n'était pas admis, il en allait autrement; et cette clause introduisait dans notre législation un principe intrinsèquement mauvais. Or, les députés étaient appelés à se prononcer sur le projet en bloc et non sur chaque clause séparément. Dès lors, et quelque recommandable que soit par ailleurs la confédération, les catholiques peuvent-ils par leur vote collaborer à introduire le divorce dans notre législation? ' (SCHEC, Rapport 1959, p. 33, note 8). 
des positions finalement corroborées par les plus éminents canonistes romains ${ }^{10}$.

Le premier évêque à intervenir est Mgr Joseph Larocque, évêque de Saint-Hyacinthe, lequel dans deux lettres à Mgr Baillargeon est d'avis que tout catholique devrait s'opposer, même au risque de faire échouer le projet de confédération, à un ordre de choses qui pourrait mettre les catholiques dans l'obligation légale de suivre des lois contraires à leur foi ${ }^{11}$. Mgr Baillargeon répond à son suffragant dans deux longues lettres, l'une du $1^{\text {er }}$ janvier 1865 et l'autre du 8 février, mais dont la substance est la même ${ }^{12}$. S'appuyant sur l'opinion des théologiens qu'il a déjà consultés, dont $M$. Taschereau sans le nommer, Mgr Baillargeon affirme ne pas partager les alarmes de son suffragant. Les députés du Bas-Canada, dans la Conférence qui a formulé le projet de constitution de la Confédération, n'avaient ni à décider si le droit de sanctionner le divorce appartenait à l'autorité souveraine de l'Etat, ni à le créer ou le constituter dans la province, puisqu'il est supposé comme un principe par la constitution de la province, qu'il y existe déjà de fait en vertu de la dite constitution et que la Chambre en a déjà fait usage plus d'une fois. Les députés n'avaient donc qu'à désigner le corps législatif auquel ce pouvoir appartiendrait sous la Confédération. C'est aussi à quoi ils se sont bornés en l'attribuant aux Chambres fédérales, et par là en le retirant aux législatures provinciales. Il ne s'agit en l'occurrence que d'un transfert de l'iniquité, de la législature provinciale à la législature fédérale. "Nous n'en serons donc pas plus mal, nous Canadiens et Catholiques - la source du mal et du scandale sera portée plus loin : nous n'en serons que mieux, à mon avis. " Mgr Baillargeon semble d'autant moins alarmé que dans sa pensée seules les questions du divorce relèveraient de la législature fédérale, tandis que celles relatives au mariage seraient laissées aux chambres particulières des Provinces. Il ajoute enfin que, même s'il devait en résulter un plus grand danger, les évêques ne peuvent rien pour faire rayer cette clause, étant donné que leurs collaborateurs dans le projet de constitution, les ministres catholiques, n'ont pas réussi à obtenir de meilleures garanties sur le même point.

On ne peut passer sous silence deux paragraphes de la lettre du 8 février, qui illustrent plus éloquemment que tout autre témoignage l'attitude de Mgr Baillargeon au cours de cette période pré-confédérative :

10 Rappelons que depuis le 14 novembre Mgr Bourget est en route pour Rome avec deux projets bien précis : la fondation d'une université catholique à Montréal et le démembrement de la paroisse Notre-Dame. D'autre part, accompagné de M. E.-A. Taschereau, Mgr Horan, évêque de Kingston, s'est lui aussi embarqué pour Rome, pour défendre la cause de l'Université Laval et appuyer les motifs de démission du Coadjuteur de Québec. Quant à la clause relative au mariage et au divorce, M. Taschereau en soumettra l'étude à ses anciens maîtres, les canonistes De Angelis et Ballerini, tandis que Mgr Horan consultera Mgr Capalti, secrétaire de la Propagande et éminent canoniste.

11 Diocèse de Saint-Hyacinthe, I, 92: Mgr Larocque à Mgr Baillargeon, 3 février 1865. L'original de la première lettre (mi-décembre 1864) a été perdu. 12 Reg. Lettres, 28, 238 et 271. 
Telle est, Monseigneur, ma manière de penser sur cette question : opinion que je soumets humblement au jugement éclairé de Votre Grandeur, en confidence; mais que je n'ose manifester, de peur de me tromper et d'induire les autres en erreur, et peut-ètre de scandalıser ceux qui ne partagent pas ma manière d'envisager les choses ${ }^{13}$.

Et cet autre paragraphe, qu'on trouve en conclusion des considérations sur l'affaire du Code Civil :

Votre Grandeur trouvera peut-être que je suis trop confiant. Vraiment, c'est un peu mon caractère; puis, je n'aime point à me plaindre sans un grave sujet, à me prononcer sans voir clairement la vérité, encore moins à condamner sans connaître, et à crier avant de sentir le mal...14.

Il faut se garder de voir dans ces dernières réflexions quelque intention de la part de Mgr Baillargeon à l'endroit de son suffragant. M. Cazeau cependant se souviendra qu'elles ont été écrites, d'autant plus que les consultations se feront désormais au niveau des grands vicaires. Pour cette raison sans doute, la voix de Mgr Baillargeon se taira sur la question de la Confédération jusqu'à son mandement de juin 1867 et c'est à regret. Ses interventions fermes, mais toutes de déférence et de pondération dans la polémique en cours sur le Code Civil, nous en auraient laissé espérer autant de lui dans l'affaire de la Confédération.

Aussitôt après Mgr J. Larocque, c'est le Grand Vicaire Truteau de Montréal qui, en l'absence de Mgr Bourget, entreprend des consultations auprès du siège métropolitain. Sa lettre du 8 février 1865 à M. Cazeau laisse percer des doutes au sujet du Code Civil, sans soulever encore la question du mariage et du divorce dans le projet de confédération. Cependant, M. Cazeau le devance et, dans sa réponse du 10 février, il abordera la question par le biais en ces termes :

Mgr l'Evêque de Saint-Hyacinthe qui avait exprimé ses alarmes à Mgr

de Tloa [Mgr Baillargeon] sur l'œuvre de la codification, a reçu ces jours derniers de la part de ce dernier une réponse qui a dû le consoler. Il a dû être aussi rassuré par le même prélat sur la question du divorce dont [on] a fait tant de bruit, et à propos de laquelle le True Witness a montré beaucoup plus de zèle que de prudence ${ }^{15}$.

M. Truteau, bien sûr, n'a pas aimé la flèche décochée contre le True Witness et le 14, février il écrit à M. Cazeau : "Je trouve qu'il s'agit ici d'une question religieuse. S'il ne s'agissait que de la politique, il serait certainement prudent de se taire; mais il y a ici plus que de la politique ${ }^{16}$. A cette lettre de M. Truteau s5en ajoute une autre de George Clerk, lequel a pris soin de consulter lui aussi les théologiens, avant de s'attaquer à la position du Courrier du Canada qui ne voyait qu'un transfert de juridiction dans l'affaire du divorce. M. Cazeau répond à ses deux correspondants le 17 février et sa position est en tout point celle déjà connue de Mgr Baillargeon. Néanmoins, la déclaration de G. E. Cartier sur l'unanimité des évêques du Bas-Canada en faveur de la Confédération, amena aussitôt $M$. Truteau à protester auprès

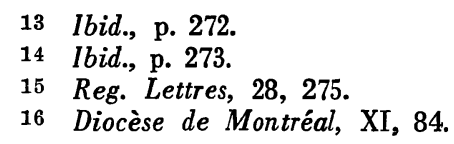

16 Diocèse de Montréal, XI, 84. 
du Grand Vicaire de Québec, lequel lui répondit en ces termes le 26 février :

En effet, Mgr de Tloa lui [G.-E. Cartier] a exprimé, ainsi qu'au premier ministre, Sir E.-P. Taché que, sans être enthousiasmé pour la Confédération, il s'y résignerait plutôt qu'à la représentation basée sur la population qui devenait inévitable. La même chose lui a été dite par Mgr Lynch, évêque de Toronto, et il croit que Mr le Grand-Vicaire Raymond, de Saint-Hyacinthe, lui a parlé dans le même sens. Quant aux autres chefs ecclésiastiques de la Province, qui n'ont pas émis d'opinion, il a sans doute regardé leur silence comme un acquiescement, et il a cru avec raison que, s'ils avaient été opposés au projet, ils n'auraient pas manqué de le proclamer d'une manière quelconque ${ }^{17}$.

M. Cazeau ne manque pas l'occasion dans cette même lettre d'exposer à nouveau et très clairement sa position quant à l'article sur le mariage et le divorce. Toutefois, la décision des canonistes romains, émise le 9 mars, devait lui servir d'argument décisif pour emporter la conviction de M. Truteau, du moins le pensait-il. Aussi n'eut-il rien de plus pressé que de la lui communiquer le 31 mars, d'autant plus qu'elle appuyait la position soutenue jusqu'ici par le siège métropolitain de Québec. Il en fallait pourtant davantage pour convaincre M. Truteau. Dans sa lettre du 3 avril, il proteste que la question a été mal posée à Rome, puisqu'on a laissé croire qu'il s'agissait d'un simple transfert de juridiction, au lieu de la création d'un nouveau pouvoir. Il ajoute même que le projet de confédération n'ira pas plus loin, étant donné la résistance qu'on lui oppose fermement dans les Maritimes. " $\mathrm{La}$ consultation de Rome ne vous a pas converti, lui répond M. Cazeau le 7 avril, mais je vais tenter un nouvel effort pour qu'il ne soit pas dit que je vous aurais laissé mourir dans l'impénitence finale ${ }^{18}$." Suit l'énumération des bills, avec rappel de date, par lesquels la Législature du Bas-Canada a accordé le divorce depuis l'Acte de 1840; et sanctionnés chaque fois par le gouvernement de Londres.

Si cette phase de controverse avait abouti à des "conversions " réelles ou présumées dans le Bas-Canada, elle devait maintenant prendre des proportions tout à fait inattendues en dehors des frontières et couvrir à la fois deux autres provinces. L'incident est déclenché par une lettre de Mgr Horan du 13 septembre à $M$. Cazeau et dans laquelle l'évêque de Kingston, encore sous l'effet d'une surprise bien compréhensible, transmet la substance d'une lettre que vient de lui adresser John A. Macdonald ${ }^{19}$. Ce dernier, se disant inquiet de l'attitude hostile des catholiques du Nouveau-Brunswick quant au projet de confédération, prie Mgr Horan d'aller les " convertir» au projet en compagnie de M. Cazeau. Mgr Horan veut d'abord connaître l'avis de ce dernier, de même que celui de Mgr Baillargeon. Si la décision était favorable, il ne pourrait lui-même entreprendre le voyage avant le début de novembre.

17 Reg. Lettres, 28, 289.

18 Ibid., p. 310.

19 Haut-Canada, XI, 138. 
La suite des événements reste assez mystérieuse, puisqu'il n'est resté aucune trace à Québec de la réponse de M. Cazeau à Mgr Horan. On sait cependant que la mission se prépare, puisque le 2 novembre G.-E. Cartier demande à M. Cazeau de conférer avec son collègue Hector Langevin, pour établir les points essentiels à mettre sous les yeux des députés catholiques du Nouveau-Brunswick et de la Nouvelle-Ecosse ${ }^{20}$. Puis, à nouveau, hiatus complet sur le déroulement des événements, si ce n'est une lettre du 30 novembre de Cartier à M. Cazeau, le remerciant, de même que Mgr Horan, des services qu'ils ont rendus à la cause de ì Confédëration dans leur voyage au îNouveau-Brunswick et en Nouvelle-Ecosse ${ }^{21}$. Cartier accuse également réception de deux importantes lettres de Cazeau, mais dont il n'est resté aucune copie à Québec.

Il est facile, au premier coup d'œil, de tirer les conclusions les plus fantaisistes de cet épisode. Cependant, à la lumière des événements survenus un peu plus tôt dans les Maritimes, on en arrive à une interprétation plus sereine. La chose nous est révélée par M. Ferdinand Gauvreau, missionnaire à Miramichi, dans une lettre à M. Cazeau du 20 mars $1865^{22}$. Dans leur opposition farouche au projet de confédération, certains ministres des Maritimes colportaient partout que les missionnaires acadiens répétaient à leurs fidèles que le clergé du BasCanada était opposé à la Confédération des provinces. La nouvelle fait son chemin jusqu'à Québec, alertant tantôt les membres de la législature, tantôt Mgr Baillargeon qui communique aussitôt avec Mgr Rogers et le prie de faire enquête. Interrogé dans cette affaire, le missionnaire Gauvreau dénonce la supercherie et fait tenir à M. Cazeau la substance de deux lettres que Gauvreau lui-même, au nom des missionnaires acadiens, a adressées à Mgr Rogers. Si donc Mgr Baillargeon ne s'est pas opposé à la mission Horan-Cazeau dans les Maritimes, on peut croire que, malgré le contexte politique de l'événement, il a choisi l'occasion, non seulement de démentir sur place la prétendue opposition du clergé du Bas-Canada à la Confédération, mais aussi de laver d'une façon qui ne laissait aucune équivoque la réputation injustement exploitée des missionnaires acadiens.

Jusqu'ici on a entendu les voix des diocèses de Saint-Hyacinthe et de Montréal, mais de Trois-Rivières on est resté sans écho. Il faut attendre au 9 décembre avant que M. Laflèche, Grand-Vicaire et futur évêque de ce diocèse, n'intervienne à son tour, dans un concours de circonstances qui laisse voir qu'il n'était peut-être pas le seul intéressé. De passage pour quelques jours à l'évêché de Montréal, il écrit de là à M. Taschereau, supérieur du Grand Séminaire de Québec, le pressant de lui adresser à Montréal copie de la décision des théologiens romains sur la clause du mariage et du divorce.

Si ma mémoire ne me fait défaut, le Courrier $d u$ Canada a reproduit ce document important, mais je ne me rappelle point sur quel $\mathrm{N}^{\circ}$ et je ne l'ai point en ce moment-ci sous la main. Attendu que j'ai des raisons

20 Gouvernement, XI, 138.

21 Ibid., p. 139.

22 Nouveau-Brunswick, III, 162. 
de me procurer discrètement cette pièce, je m'adresse avec confiance à vous à cet effet, et si vous me le permettez, je vous demanderais votre opinion personnelle sur la conformité de ce vote avec les principes catholiques, en vous donnant l'assurance d'en user avec toute la prudence convenable 23 .

Il est évident que la décision de Rome rencontrait toujours des opposants à Montréal et on tentait vraisemblablement de vérifier par cette démarche si la position de Québec n'avait pas évolué depuis. Dans sa réponse du 11 décembre, contenue en huit pages, M. Taschereau relève les accusations qu'on a portées contre lui sur la façon dont il avait posé la question à Rome, puis il ajoute : "J'ai exposé les faits tels que je les comprends et plus je les étudie, plus je crois n'y avoir commis aucune erreur... Vous me demandez mon opinion personnelle sur la conformité du vote en faveur de la Confédération avec les principes catholiques. Je n'en doutais nullement avant d'avoir consulté, et vous comprenez que je suis plus convaincu que jamais ${ }^{24}$."

A l'issue de cette deuxième phase, on sent que la nouvelle constitution est de plus en plus une chose acquise. Les évêques de la province ecclésiastique de Québec, appelés à se prononcer le 29 juillet sur l'érection d'une nouvelle province ecclésiastique dans le Haut-Canada, jugent que le temps n'est pas encore venu de diviser la province ecclésiastique de Québec et qu'il faut attendre pour cela que les changements qui se préparent dans l'état civil et dans le gouvernement du Canada soient accomplis ${ }^{25}$.

Toutefois, l'incident du Bill sur l'éducation, accordant des privilèges aux minorités protestantes du Bas-Canada sans octroyer les mêmes droits aux minorités catholiques du Haut-Canada, avait fortement ébranlé la confiance du clergé et de l'épiscopat à l'endroit de G.-E. Cartier et d'Hector Langevin. Les évêques avaient réagi auprès des plus hautes autorités civiles et Mgr Bourget, dans sa démarche auprès de Mgr Baillargeon pour rendre publique la pétition des évêques, terminait en ces termes sa lettre du 3 août : "Il est facile de conclure que le Ministère veut la Confédération à tout prix; mais, s'il en est ainsi, il ne sera pas béni ${ }^{26}$. » Le même jour, M. Cazeau réprimande sévèrement Cartier et l'enjoint de racheter sa pusillanimité, autrement \& c'est le suicide de votre Ministère ». M. Cazeau est d'autant plus survolté que Cartier l'avait assuré peu auparavant que "les catholiques des provinces maritimes pouvaient compter sur la législature fédérale où toutes les minorités auraient protection $^{27}$ ». On sait que Cartier retira aussitôt le projet de loi, provoquant ainsi l'éloignement de Galt. M. Cazeau le félicita de son geste et lui fit cette dernière recommandation :

Si vous voulez conserver la confiance des honnêtes gens de votre origine, il faut que, lorsque vous serez en Angleterre, vous montriez le plus vif intérêt à faire accorder aux catholiques les mêmes garanties, droits et

23 Dioc. de Québec, IV, 160.

24 Archives Séminaire Trois-Rivières, T. Al-99-02.

25 Reg. Lettres, 28, 560.

26 Diocèse de Montréal, XI, 97.

27 Vicaires généraux, XVI, 151. 
privilèges qu'aux protestants. Tous, j'en suis sûr, auront les yeux fixés sur vous et vous tiendront pour responsable de ce qui sera réglé, de quelque manière que ce soit 28 .

Pendant que Cartier et ses collègues voguent vers l'Angleterre, on assiste ici à la phase finale de cette période pré-confédérative. A l'archevêché de Québec, si l'atmosphère n'est pas à l'exubérance, elle n'est pas non plus à la résignation dépitée. C'est un climat d'acceptation, positive et optimiste, et dont on connaît déjà les motifs. Entre temps, deux nouvelles figures sont venues joindre les rangs de l'épisconat : Mgr Laflèche a été fait coadjuteur de Mgr Cooke le 20 novembre 1866 et Mgr Langevin a été élu le 15 janvier 1867 premier évêque du diocèse nouvellement créé de Rimouski. Mgr Charles Larocque occupe déjà le siège de Saint-Hyacinthe depuis juillet 1866.

Le 19 février 1867, M. Cazeau répond à l'un de ses amis de vieille date, le Révérend O'Brien, devenu, depuis son départ du Canada, GrandVicaire de Simerick en Irlande, qui lui demande son opinion sur la Confédération. Ce n'est pas tant la position de Cazeau, trop condensée dans cette lettre, qui intéresse que l'attitude de l'épiscopat des Maritimes. M. Cazeau l'informe donc que l'archevêque Connolly d'Halifax, de même que ses collègues d'Arichat et de Chatham, sont favorables, mais que l'évêque de Saint-Jean, N.-B. y est opposé ${ }^{29}$.

C'est à la mi-mars 1867 que la position du siège métropolitain de Québec se trouvera le plus clairement définie. On doit le document à la plume de M. Cazeau, répondant à une lettre de George Clerk, lequel de son côté vient de soumettre à Mgr Baillargeon et à son Grand-Vicaire la position qu'il entend suivre dans le True Witness vis-à-vis la Confédération, qui sera bientôt un fait accompli.

Sa grandeur [Mgr Baillargeon] croit que le plan que vous vous êtes tracé au sujet de la Confédération est le plus sage. Maintenant que cette mesure est un fait accompli, le devoir de tout bon citoyen est d'en tirer le meilleur parti possible, surtout s'il tient à demeurer sujet britannique.

I ne faut pas oublier que lorsque la question de la Confédération a été ouverte, tout gouvernement, sous la constitution actuelle, était devenu une impossibilité, les deux partis dans l'assemblée législative se trouvant à peu près d'égale force. On était menacé de la représentation basée sur la population, que les protestants de nos townships n'avaient pas de répugnance à accepter. La dissolution de l'Union était impraticable, elle présentait des difficultés immenses pour le règlement de la dette, du tarif, des canaux, etc., et d'ailleurs on n'avait pas de majorité pour la voter. Il ne restait plus, pour sortir d'embarras, que deux alternatives : ou la confédération, ou l'annexion aux Etats-Unis. Nos hommes d'état en qui nous avions le plus de confiance se prononcèrent pour la confédération, afin d'éviter l'annexion. De ce côté se trouvaient des hommes comme Sir E.P. Taché, MM. Cartier, Cauchon, Langevin, Chapais et, en dehors de la politique, des hommes qui n'inspiraient pas moins de confiance, tels que le juge Morin, homme d'un patriotisme si intègre et éclairé et généralement tous les amis de la religion et de l'ordre. De l'autre côté, que voyait-on? Des hommes visant à l'annexion et consi-

28 Ibid., p. 152.

29 Ibid., p. 154. 
dérés, par leurs antécédents, comme assez peu favorables à la bonne cause et dont le clergé n'avait guère à se louer.

Dans un pareil état de choses, il n'y avait pas pour le clergé à balancer. Il était naturel que ses sympathies fussent plutôt pour ses défenseurs que pour ses détracteurs, pour ceux qui voulaient nous conserver à la domination de l'Angleterre plutôt que pour ceux qui voulaient nous engouffrer dans celle de la république voisine. J'ignore quelle était l'opinion dans le diocèse de Montréal, mais ici il y avait à peu près unanimité. Sans doute personne n'était enthousiasmé de la confédération, mais elle était acceptée comme un moindre mal que l'annexion. Le clergé, comme corps, ne s'est prononcé ni pour ni contre la confédération et, s'il penchait davantage du côté où se trouvaient ses amis, il n'a fait aucune démarche pour exprimer son opinion. S'il avait vu des dangers sérieux pour la religion dans le nouveau projet de constitution, il aurait protesté comme il le fit lors de l'union des deux provinces. Mais en face de l'annexion qui présentait des dangers autrement graves, il n'avait rien de mieux à faire que de garder le silence et de laisser faire les membres honorables à qui incombait la pénible tâche de préparer la confédération.

Que ceux-ci aient fait tous leurs efforts pour rendre la mesure aussi équitable que possible en faveur de leur pays, c'est ce dont nous ne pouvons pas douter. Mais, comme vous le remarquez dans votre lettre, ils n'étaient pas les seuls maîtres de la situation; il fallait écouter les prétentions des hommes d'état des autres provinces, avoir recours à des compromis, marchander, etc. Tout porte à croire qu'ils ont fait noblement leur devoir, si on en juge par la résistance qu'ils ont faite avec succès aux prétentions des protestants du Bas-Canada, qui voulaient se faire garantir par la nouvelle constitution des droits et privilèges que n'ont pas les catholiques du Haut-Canada.

Quoiqu'il en soit, je crois que si nous ne nous sommes pas toujours entendus tous deux sur la question de la confédération, nos idées là-dessus paraissent maintenant tout à fait identiques. Vous comprenez que je n'en trouverai le True Witness que plus aimable ${ }^{30}$.

On reconnaît déjà dans ce document la substance de certains paragraphes du mandement à venir de Mgr Baillargeon. Et à comparer cette lettre avec la réponse faite au même correspondant le $1^{\text {er }}$ juillet 1864 , on se rend compte que la position du siège de Québec est restée en tout point identique à ce qu'elle était au début de cette période pré-confédérative.

\section{AU LENDEMAIN DE LA PROCLAMATION}

Tout laisse croire qu'après la proclamation de la constitution Mgr Baillargeon n'envisageait de poser aucun geste significatif pour souligner l'événement. Ce fut Mgr Laflèche qui proposa à son métropolitain que chaque évêque émette un mandement particulier à cette occasion. La suggestion du coadjuteur de Trois-Rivières fut accueillie avec enthousiasme par Mgr Baillargeon, qui accusa réception de son "intéressante et véritablement importante lettre du 8 de ce mois [mai] ${ }^{31}$ ». Du 10 au 14. mai, Mgr Baillargeon eut tôt fait de recueillir l'avis des évêques de Kingston, de Hamilton et de Rimouski ${ }^{32}$. Tous se disaient favorables à la suggestion, ajoutant qu'il faudrait que le mandement de chaque

30 lbid., p. 155.

31 Reg. Lettres, 29, 24. L'original de la lettre de Mgr Laflèche a été perdu.

32 Ces évêques se trouvaient à Québec vraisemblablement pour suivre les exercices spirituels de leur retraite annuelle en compagnie de Mgr Baillargeon. 
évêque fut préparé à l'avance, afin d'être publié aussitôt après la proclamation de la constitution. Quant aux évêques du Haut-Canada, ils estimaient qu'ils n'avaient pas besoin de publier un tel mandement dans leurs diocèses, où il n'y avait aucune opposition à la Confédération. Cependant, les sentiments étant autres dans le Bas-Canada, les évêques étaient conscients les premiers de la portée d'un geste de solidarité de leur part. Voilà pourquoi Mgr Baillargeon ajoute dans sa lettre :

Mais il conviendrait certainement que ceux du Bas-Canada s'entendissent tous et s'accordassent à publier chacun leur mandement dans le même but sur ie sujer en question. Ii faudrait donc engager l'Evêque de Montréal et celui de Saint-Hyacinthe. Or personne ne saurait mieux faire la chose que Votre Grandeur qui a le mérite d'avoir suggéré le dit mandement. Je vous prie donc de leur écrire à cette fin. Pour l'Evêque de Rimouski, vous pouvez le regarder comme prêt à se joindre à vous. Il est plein de zèle pour cette bonne ouvre; il sera donc prêt, aussi bien que votre [dévoué serviteur] 33 .

Si l'on ignore comment s'est effectué le sondage d'opinion dans les jours qui ont suivi, on sait que Mgr Bourget de son côté n'a pas attendu de respecter la consigne à l'effet que les évêques s'entendissent à publier leur mandement dans le même but. Dès le 25 mai, l'évêque de Montréal adressait à son clergé une circulaire sur la conduite à tenir pendant les prochaines élections ${ }^{34}$.

Entre temps, Mgr Laflèche s'était mis activement à la rédaction du mandement qui devait porter la signature de Mgr Cooke et s'était empressé de le soumettre à Mgr Baillargeon. Le 21 mai, ce dernier lui répond :

J'ai lu avec un vif intérêt votre belle lettre pastorale que j'ai l'honneur de vous renvoyer ci-jointe. Je ne partage pas du tout l'opinion de Mgr Cooke "qui la trouve trop politique ", il me semble qu'elle dit fort bien ce qu'il faut dire et ce que vous avez voulu dire. Je n'ai donc aucune correction ni aucun changement à vous suggérer. Au reste, Votre Grandeur a le temps de la revoir et d'y ajouter ou d'y retrancher ce qu'elle jugera à propos. Je voudrais bien que la mienne fût faite. C'est une grande affaire pour moi que d'écrire sur ce sujet auquel je suis fort étranger 35 .

33 Ibid.

34 Mandements des Evêques de Montréal, vol. IV, pp. 212-214.

35 Reg. Lettres, 29, 27. - Mgr Charles Larocque, à l'exemple de Mgr Laflèche, aurait transmis à Mgr Baillargeon le 13 juin son projet de mandement avant de le publier. D'autre part, Mgr Bourget aurait déclaré dans une lettre du 15 octobre à Mgr Charles Larocque que, lors d'une rencontre à Berthier, l'archevêque de Québec avait approuvé sa "manière de procéder " jusqu'alors. On relève ces faits intéressants dans W. Ullman, The Quebec Bishops and Confederation (Can. Hist. Rev., sept. 1963, p. 231); de plus, l'assertion de Mgr Bourget est corroborée par le R.P. Léon Pouliot, S.J. dans son étude déjà citée (p. 40). Les archives de l'archevêché de Québec sont muettes sur ces relations des deux évêques avec Mgr Baillargeon.

Quant au projet de mandement collectif sur les élections et les mauvais journaux, c'est le 10 novembre suivant qu'il fut proposé par les évêques de Montréal et de Trois-Rivières, lors d'une réunion de l'épiscopat à Toronto. Mgr Laflèche en avait déjà préparé une première rédaction quand Mgr Baillargeon, devenu archevêque de Québec aux derniers jours d'août, l'informa le 7 décembre qu'il fallait \& plutôt attendre l'époque du prochain concile [provincial] pour publier un document de cette importance, après l'avoir travaillé et discuté en commun., (Reg. Lettres, 29, 111). 
La lettre pastorale de Mgr Baillargeon porte la date du 12 juin et le Courrier du Canada la publia avec celle de Mgr Cooke dans son édition du 17 juin. Le document, qui est à la fois un mandement sur la Confédération et une circulaire sur les élections, s'adresse non seulement au clergé mais à tous les fidèles de l'archidiocèse. Il faut faire effort pour arriver à distinguer les deux parties, assez imbriquées, de cette lettre pastorale, dont la composition laborieuse s'alourdit au surplus de surcharges et de redites. A y regarder de près, on se rend compte que les subtilités de certains paragraphes traduisent davantage qu'un simple rappel de soumission à l'ordre établi. En effet, si l'auteur appuie largement sur la valeur sacrée de l'autorité et sur l'obligation stricte qui incombe à tout citoyen de s'y soumettre, il ne considère pas moins, quoique plus discrètement, les avantages certains de la nouvelle constitution, tant pour les individus que pour la prospérité commune.

Ce qui doit nous rassurer, N.T.C.F., c'est que la nouvelle forme de gouvernement qui vient de nous être donnée, a été préparée avec soin par des hommes bien connus, eux aussi, par leur patriotisme, aussi bien que par les services qu'ils ont rendu à leur commune patrie. Si elle n'est pas sans défauts, si elle n'est pas tout ce qu'on aurait pu désirer qu'elle fût, rappelons-nous que rien n'est parfait dans ce monde, et que, dans un pays comme le nôtre, où tant d'intérêts divers sont en présence, il était impossible de se refuser à de mutuelles concessions, et d'arriver à un arrangement qui pût donner satisfaction à tout le monde...

Au reste, n'oublions pas, N.T.C.F., combien nous avons à nous féliciter de vivre sous l'égide de l'Empire Britannique. Il est peu de pays au monde qui ait marché aussi rapidement que le nôtre dans la voie du véritable progrès, et nous n'en connaissons aucun ou la religion jouisse d'une plus grande liberté, et exerce une plus large part d'influence. Tout cela est dû, après la protection du ciel, à la politique éclairée des hommes d'Etat qui, depuis un quart de siècle surtout, président aux destinées de la mère-patrie ${ }^{36}$.

Comme on le voit, deux paragraphes sont consacrés à définir ce qui, dans la pensée des chefs religieux de Québec, est toujours apparu comme le premier mérite de la nouvelle constitution, celui de conjurer le danger d'annexion. Il n'y a pas à s'étonner que la lettre pastorale prenne position dans la ligne déjà tracée par M. Cazeau dans sa lettre à Clerk du 16 mars 1867.

Cependant, la Confédération n'a été possible qu'après de mutuelles concessions et au prix de certains risques pour l'avenir. Bien conscient de ces éventualités, Mgr Baillargeon écrit :

C'est aux hommes à qui vous allez confier le soin de vous représenter dans l'un et l'autre parlement, de s'unir fortement ensemble pour conjurer le danger, s'il existe, et pour tirer le meilleur parti possible de la situation. Vous avez donc une raison de plus de les choisir parmi ceux qui se distinguent par leur honnêteté, leur énergie et leur dévouement à la chose publique... Vous vous garderez de donner vos voix à des hommes disposés à la combattre [la constitution] ou à mettre des entraves à son fonctionnement, mais vous les donnerez à des citoyens éprouvés et reconnus comme ayant à cœur de la faire servir au plus grand bien du pays ${ }^{37}$.

36 Mandements des Evêques de Québec, vol. 4, p. 581.

37 Ibid. 
Ces considérations s'inscrivaient naturellement dans la prise de position du mandement. Si la prudence en face d'un avenir incertain exigeait davantage qu'une simple mise en garde contre les désordres possibles en période électorale, pareilles considérations réduisaient sensiblement les chances des candidats qui envisageaient de se faire élire sous un autre étendard.

Telle a été l'option que le siège métropolitain de Québec a maintenue et défendue au cours des événements qui ont donné naissance à la Confédération. Se rontenter d'affirmer que ses chefs religieux se sont soumis avec résignation à la Confédération, c'est rester en deçà de la réalité. A tout prendre, c'est à se demander s'ils n'ont pas fait encore plus que de la désirer.

Armand GaGNÉ, ptre, Archiviste à l'Archevêché de Québec. 